\title{
N uevas alternativas en el tratamiento del cancer gástrico avanzado
}

\author{
Marcelo G arrido, G eraldine Melgoza, H éctor $\mathbf{G}$ alindo, \\ Jorge Madrid, César Sánchez, Bruno Nervi, Manuel \\ Alvarez, Eric 0 rellana.
}

\section{Treatment of advanced gastric cancer with oxaliplatin plus 5-fluorouracil/ leucovorin (FO LFOX-4 chemotherapy)}

Background: Chemotherapy improves survival in advanced gastric cancer. However the most active combinations have a high level of toxicity that limits their use. Aim: To assess the response, toxicity and survival of patients with advanced gastric cancer, treated with oxaliplatin plus 5-fluorouracil/leucovorin (FOLFOX-4 chemotherapy). Material and methods: Patients with stage IV gastric cancer, according to the American Joint Committee on Cancer or with relapsed disease and functional capacity 0-2 of the South West Oncology Group, were included. FOLFOX-4 chemotherapy was used as first or second line treatment. The response to treatment and survival were assessed. Results: Between 2003 and 2006, 29 patients (median age 52.5 years, 69\% males) were treated. FOLFOX-4 was given as first line treatment in $65 \%$ patients and as second line in $35 \%$. There was a complete response in $4.6 \%$, partial response in $68 \%$, stable disease in $20.6 \%$ and progression in $6.8 \%$. Toxicity was observed in $51 \%$ of patients, that was hematological and non hematological grade $3 / 4$ in $14 \%$. Median survival was 12.5 months. Conclusions: FOLFOX-4 chemotherapy was active in advanced gastric cancer and had a low level of toxicity (Rev Méd Chile 2007; 135: 1380-7).

(Key words: Antineoplastic combined chemotherapy protocols; Organo platinum compounds; Stomach neoplasms)

Recibido el 11 de abril, 2007. Aceptado el 28 de junio, 2007.

Departamento de Hematología-Oncología, Centro de Cáncer, Pontificia Universidad Católica de Chile, Santiago de Chile.

$\mathrm{E}^{1}$ láncer gástrico (CG) es el segundo tumor maligno más frecuente y una de las principales causas de muerte por cáncer en el mundo ${ }^{1}$, en

Correspondencia a: Eric Orellana Ulunque. Centro de Cáncer, Pontificia Universidad Católica de Chile. Diagonal Paraguay 319, Santiago, Chile. Fono: 56-2-3546919. Fax: 56-2-2472327. E mail: eric@med.puc.cl
Chile representa la primera causa de mortalidad por neoplasia maligna ${ }^{2}$.

Es un tumor agresivo que se presenta en el $65 \%$ de los casos en forma avanzada (T3 o T4) y en $85 \%$ con ganglios comprometidos. La sobrevida global (SG) a 5 años de los pacientes con enfermedad resecada es de 30\% y en enfermedad avanzada la sobrevida media sin tratamiento es de 5,4 meses $^{3}$. 
En enfermedad metastásica la quimioterapia (QMT) basada en 5-fluorouracilo (5-FU) ha demostrado prolongar la sobrevida y mejorar la calidad de vida en relación al mejor soporte paliativo $^{4}$ y metaanálisis han demostrado una reducción en la mortalidad de $61 \% \%^{5}$.

Varios estudios randomizados han evaluado la superioridad de un régimen de quimioterapia sobre otro, logrando tasas de respuesta entre 8,6\% y $45 \%$ con medianas de SG entre 5 y 9,4 meses, de ellos el esquema que incluye irinotecan se presentó en el Congreso de la American Society of Oncology (ASCO) y aún no ha sido publicado ${ }^{6-14}$.

Las comparaciones entre las quimioterapias más activas han mostrado tasas de respuesta global de 46\% (95\% IC, 37-55\%) con FEC (5-FU, epirrubicina y cisplatino) y $21 \%$ (95\% IC, $13-28 \%)$ con FAMTX (5-FU, adriamicina y metotrexato) (p $=0,00003$ ), con sobrevida mediana de 8,7 y 6,1 meses ( $\mathrm{p}=0,0005)$ y SG a 2 años de $14 \%$ (95\% IC, $8-20 \%)$ y $5 \%$ (95\% IC, $2-10 \%)(\mathrm{p}=0,03)$, respectivamente ${ }^{10}$.

El estudio fase III V325 que comparó DCF (docetaxel, cisplatino y 5-FU) con FC (5-FU y cisplatino) demostró que el esquema con docetaxel tenía una tasa de respuesta, sobrevida libre de eventos y SG superior, pero presentó una mayor incidencia de toxicidad hematológica con neutropenia, neutropenia febril e infección neutropénica de $84 \%, 16 \%$ y $14 \%$, respectivamente ${ }^{14}$.

Debido al limitado beneficio y la alta toxicidad de los esquemas más activos en CG es necesaria la búsqueda de nuevos protocolos de QMT. Como agentes únicos existen varias drogas con buena actividad como irinotecan, S-1, paclitaxel y oxaliplatino con respuestas entre $18 \%$ y $45 \%$ y medianas de sobrevida entre 8,3 y 11,5 meses. Combinaciones de drogas en estudios fase II como irinotecancisplatino, irinotecan-5-fluorouracilo/leucovorina, S-1-irinotecan, oxaliplatino-5-fluorouracilo/leucovorina han mostrado respuestas de 40\%-59\% con sobrevida mediana de 9 a 12 meses ${ }^{15}$.

El esquema FOLFOX-4 ampliamente usado en cáncer de colon, se ha utilizado en un estudio fase II en cáncer gástrico mostrando una respuesta global de 38\%, enfermedad estable de 36\%, toxicidad hematológica grado $3 / 4$ 36\% y neuropatía periférica grado 3 de $5 \%{ }^{16}$. Además en pacientes refractarios a 5-fluorouracilo o cisplatino este esquema ha mostrado respuestas de $26 \%$, sobrevi- da mediana de 7,3 meses, toxicidad hematológica grado 2 y neuropatía periférica grado $2^{17}$.

Nuestro objetivo fue evaluar la respuesta, toxicidad y sobrevida de pacientes con cáncer gástrico avanzado tratados con FOLFOX 4 en primera o segunda línea.

\section{PACIENTES Y MÉTODOS}

Fueron incluidos pacientes con adenocarcinoma gástrico o gastroesofágico irresecable o metastásico, confirmado histológicamente, con lesiones tumorales medibles por tomografía axial computada (TAC) o cintigrama óseo, mayores de 18 años, en etapa IV de la AJCC (American Joint Committee on Cancer) o enfermedad recurrente, capacidad funcional 0-2 de la SWOG (South West Oncology Group); con función renal, medular, hepática, cardiaca y pulmonar normal y estado nutricional normal. Fueron tratados en los Departamentos de Cirugía Digestiva y HematoOncología de la Pontificia Universidad Católica de Chile, entre noviembre de 2003 y junio de 2006. Se incluyó a pacientes con recaída de enfermedad, sometidos a cirugía, radioterapia o quimioterapia excepto que contraindicara el uso de FOLFOX 4 en primera o segunda línea. Se excluyeron a pacientes con embarazo o lactancia en curso, con otro cáncer en tratamiento, excepto cáncer de piel no melanoma y carcinoma in situ de cérvix y aquellos pacientes que no desearan expresamente someterse a esta QMT o que tuvieran contraindicaciones desde el punto de vista psiquiátrico.

Se usó quimioterapia con FOLFOX-4 en primera o segunda línea administrada cada dos semanas y consistía en oxaliplatino $85 \mathrm{mg} / \mathrm{m}^{2}$ iv el día 1 , leucovorina $200 \mathrm{mg} / \mathrm{m}^{2}$ iv el día 1 y 2 , y 5 -FU 400 $\mathrm{mg} / \mathrm{m}^{2}$ iv en bolo y $600 \mathrm{mg} / \mathrm{m}^{2}$ en infusión continua de 22 horas durante día 1 y 2 .

El estado basal de la enfermedad se evaluó con TAC de tórax, abdomen, pelvis (TAC-TAP) y cintigrama óseo; la evaluación de la respuesta se realizó por criterios de la Organización Mundial de la Salud (OMS) con TAC de tórax abdomen y pelvis o cintigrama óseo según corresponda cada 8 semanas. La medición de toxicidad se realizó según los criterios del NCI (National Cancer Institute).

Por el método de Kaplan-Meier se evaluó la SG desde el diagnóstico hasta la muerte por cualquier causa. 


\section{Resultados}

Fueron incluidos 29 pacientes entre noviembre de 2003 y junio de 2006, 69\% hombres, con edad promedio de 52,5 años. Los pacientes tenían antecedentes familiares de cáncer en 34\% de los casos (gástrico 5, mama 4, esófago 2, pulmón 2) y antecedentes de cáncer gástrico en familiares de primer o segundo grado en 17\% (5 pacientes).

El 48\% de los pacientes había recaído después de haber sido sometido a cirugía, de ellos 10 casos habían recibido cirugía curativa y 4 casos cirugía paliativa.

El intervalo entre inicio de síntomas y diagnóstico fue de 3,7 meses con un rango entre 1 y 12 meses. Al diagnóstico el tumor se ubicaba en el tercio superior en 37\% (2 gastro-esofágicos), en el tercio medio $20 \%$ y en el tercio inferior 27\%, había $16 \%$ de los pacientes con compromiso difuso del estómago.
El sitio principal de metástasis fue hígado en 15 pacientes, pulmonar o pleural en 2 , óseas en 3 y peritoneo en 10 casos.

La respuesta a la quimioterapia con FOLFOX-4 en primera línea observada a las 8 semanas de tratamiento en 19 pacientes fue: respuesta completa 5\%, respuesta parcial 73\%, enfermedad estable $11 \%$ y progresión en 11\%. En 10 pacientes con FOLFOX-4 en segunda línea de tratamiento fue: respuesta parcial de 60\% y enfermedad estable en $40 \%$. La tasa global de respuesta fue de: $72 \%$ y la tasa de respuesta global más enfermedad estable fue de $92 \%$. El promedio de duración de la respuesta en el total de pacientes fue de 5,5 meses, posterior a lo cual todos los pacientes tuvieron progresión y fueron sometidos a otras líneas de quimioterapia o cuidados paliativos según correspondiera (Tabla 1) (Figura 1).

La toxicidad observada fue grado 1 en 15 pacientes (51\%) fundamentalmente neuropatía y

\section{Tabla 1. Respuesta a la quimioterapia con FO LFOX-4 en pacientes con adenocarcinoma gástrico observada en primera y segunda línea}

\begin{tabular}{|cccccccc|}
\hline \multicolumn{3}{|c}{ Respuesta en primera línea (\%) } & \multicolumn{3}{c|}{$\begin{array}{c}\text { Respuesta en segunda línea (\%) } \\
\text { N: } 10\end{array}$} \\
\hline RC & RP & EE & P & RC & RP & EE & P \\
5 & 73 & 11 & 11 & 0 & 60 & 40 & 0 \\
\hline
\end{tabular}

RC: Respuesta completa. RP: Respuesta parcial. EE: Enfermedad estable. P: Progresión.

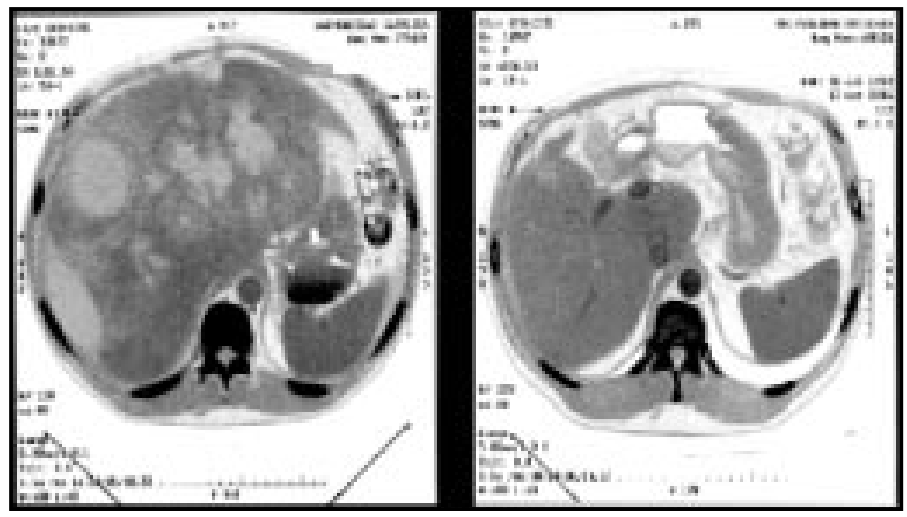

Figura 1. Tomografía axial computada de un paciente con cáncer gástrico tratado con FOLFOX-4, la imagen A corresponde al diagnóstico y la imagen $\mathrm{B}$ a la realizada como parte de la segunda evaluación a la semana 16 de tratamiento. 
toxicidad hematológica, grado 2 en 8 pacientes (27\%) especialmente neurológica y reacciones de hipersensibilidad, grado 3 en 4 pacientes (14\%) neurológica y hematológica; no hubo pacientes con toxicidad grado 4 (Tabla 2).

La SG estimada por el método de KaplanMeier fue de 12,5 meses (Figura 2).

\section{DisCUSIÓN}

El pronóstico de los pacientes con CG avanzado es pobre a pesar del desarrollo de nuevas modalidades terapéuticas. En estudios fase III con distintos regímenes de QMT, las tasas de respuesta oscilan entre $8,6 \%$ y $45 \%$ con una SG que no excede los 9,4 meses (6-14) (Tabla 3). La toxicidad hematológica grado $3 / 4$ de los protocolos que demostraron beneficio en SG fue de $32 \%$ y $84 \%$ (6,8-14) (Tabla 4). Estos estudios tienen importantes limitaciones metodológicas, algunos un número de pacientes insuficiente para demostrar efecto en la SG, no han evaluado calidad de vida exhaustivamente y los esquemas más activos como FEC y DCF tienen una toxicidad considerable que limita su uso. Es por ello que esquemas de tratamiento más efectivos y menos tóxicos son necesarios.

Los esquemas de QMT que han sido evaluados en estudios fase II en CG avanzado han mostrado

Tabla 2. Toxicidad observada en pacientes con adenocarcinoma gástrico tratados con quimioterapia FO LFOX-4 expresada en grados según clasificación del N CI

\begin{tabular}{|lcccc|}
\hline Toxicidad & Grado 1 & Grado 2 & Grado 3 & Grado 4 \\
\hline Hematológica & 4 & 0 & 2 & 0 \\
Gastrointestinal & 1 & 2 & 0 & 0 \\
Neuropatía sensorial & 8 & 3 & 2 & 0 \\
Hepática & 0 & 0 & 0 & 0 \\
Cardiaca & 0 & 0 & 0 & 0 \\
Renal & 0 & 0 & 0 & 0 \\
Cutánea & 0 & 1 & 0 & 0 \\
Hipersensibilidad & 0 & 2 & 0 & 0 \\
Fatiga & 2 & 0 & 0 \\
\hline
\end{tabular}

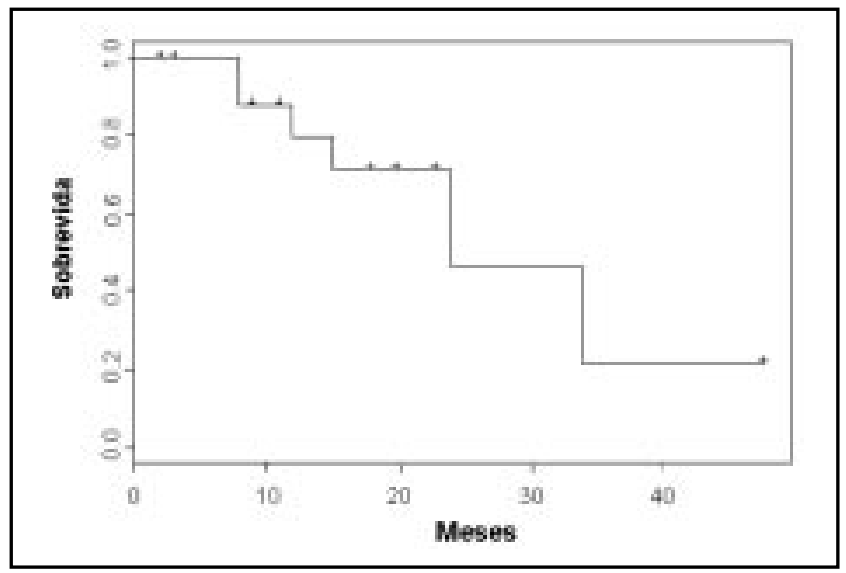

Figura 2. Curva de sobrevida de pacientes con adenocarcinoma gástrico avanzado tratados con FOLFOX-4 en primera y segunda línea. 
Tabla 3. Estudios randomizados que comparan esquemas de Q M $T$ en cáncer gástrico avanzado

\begin{tabular}{|c|c|c|c|c|c|c|c|c|}
\hline Año & Revista & Autor & \multicolumn{2}{|c|}{$\begin{array}{l}\mathrm{N} \text { por cada } \\
\text { rama de tto. }\end{array}$} & $\begin{array}{c}\text { Respuesta } \\
(\%)\end{array}$ & $\begin{array}{l}\text { SLE } \\
(\mathrm{m})\end{array}$ & $\begin{array}{l}\text { SG } \\
(\mathrm{m})\end{array}$ & $\mathrm{P}$ en $\mathrm{SG}$ \\
\hline \multirow[t]{2}{*}{1991} & $\mathrm{JCO}^{6}$ & Wils & FAMc & NR & 9 & NR & 7,2 & 0,004 \\
\hline & & & FAMTX & NR & 41 & & 10,5 & \\
\hline \multirow[t]{3}{*}{1993} & Cancer $^{7}$ & Kim & $\mathrm{FP}$ & 103 & 51 & 5,45 & NR & $>0,05$ \\
\hline & & & FA Mc & 98 & 25 & 3 & & (SLE) \\
\hline & & & $\mathrm{F}$ & 94 & 26 & 2,27 & & \\
\hline \multirow[t]{2}{*}{1998} & Cancer $^{8}$ & Icli & EEP & 64 & 20,3 & 6 & 6 & $>0,05$ \\
\hline & & & FEC & 67 & 15,3 & 7 & 5 & \\
\hline \multirow[t]{2}{*}{2002} & Ann Oncol $^{9}$ & Tebbutt & PVIF & 125 & 16,1 & 3,9 & 6,3 & 1 \\
\hline & & & PVIF Mc & 129 & 19,1 & 3,8 & 5,3 & \\
\hline \multirow[t]{2}{*}{1997} & $\mathrm{JCO}^{10}$ & Webb & FEC & 126 & 45 & 7,4 & 8,9 & 0,0009 \\
\hline & & & FAMTX & 130 & 21 & 3,4 & 5,7 & \\
\hline \multirow[t]{3}{*}{2000} & $\mathrm{JCO}^{11}$ & Vanhoefer & ELF & 132 & 9 & 3,3 & 7,2 & 0,73 \\
\hline & & & FP & 134 & 20 & 4,1 & 7,2 & \\
\hline & & & FAMTX & 133 & 12 & 3,3 & 6,7 & \\
\hline \multirow[t]{2}{*}{2002} & $\mathrm{JCO}^{12}$ & Ross & FEC & 289 & 42,4 & 7 & 9,4 & 0,315 \\
\hline & & & $\mathrm{McCF}$ & 285 & 44,1 & 7 & 8,7 & \\
\hline \multirow[t]{3}{*}{2003} & $\mathrm{JCO}^{13}$ & Ohtsu & $\mathrm{F}$ & 105 & 11,4 & 1,9 & 7,1 & 0,34 \\
\hline & & & FP & 105 & 34 & 3,9 & 7,3 & \\
\hline & & & UFTMc & 70 & 8,6 & 2,4 & 6,0 & \\
\hline \multirow[t]{2}{*}{2005} & Proc Am Soc & Dank & FLI & 170 & NR & 5 & NR & 0,088 \\
\hline & Clin Oncol & & $\mathrm{FP}$ & 163 & & 4,2 & & (SLE) \\
\hline \multirow[t]{2}{*}{2006} & $\mathrm{JCO}^{14}$ & Van Cutsem & DCF & 227 & 37 & 5,6 & 9,2 & 0,02 \\
\hline & & & $\mathrm{FP}$ & 230 & 25 & 3,7 & 8,6 & \\
\hline
\end{tabular}

FAMc $\quad:$ 5-FU, adriamicina, mitomicina $\mathrm{C}$

FAMTX : 5-FU, adriamicina, metotrexato

FP : 5-FU, cisplatino

$\mathrm{F}:$ : 5-FU

EEP : Etoposido, epirrubicina, cisplatino

FEC : 5-FU, epirrubicina, cisplatino

SLE : Sobrevida libre de eventos

NR : No registrado

respuestas entre $40 \%$ y $76 \%$ con una mediana de sobrevida entre 9 y 17 meses, de ellos los esquemas que combinan oxaliplatino con fluorouracilo y leucovorina alcanzaron altas tasas de respuesta y baja toxicidad. El protocolo FOLFOX$6^{18}$ mostró respuesta en $44,9 \%$ de los pacientes con una toxicidad hematológica grado $3 / 4$ de $38 \%$ y neuropatía grado 3 en $21 \%$. El esquema FLO $^{19}$ reportó respuestas en $43 \%$ con aun menos toxicidad hematológica grado 3/4 (4,9\%) que lo hace un esquema atractivo en este tipo de patologías.
PVIF : 5-FU en infusión continua

PVIFMc : 5-FU en infusión continua, mitomicina $\mathrm{C}$

McCF : Mitomicina C, 5-FU, cisplatino

UFTMc : Uracilo tegafur, mitomicina $\mathrm{C}$

DCF : Docetaxel, 5-FU, cisplatino

FLI : 5-FU, leucovorina, irinotecan

SG : Sobrevida global
En el mundo y en nuestro medio, el esquema de QMT FOLFOX-4 es ampliamente usado en cáncer colorrectal (CC), debido a que este protocolo mostró tasas de respuesta entre 50\% y 60\%, con prolongación de la sobrevida en comparación a 5-FU y leucovorina ${ }^{20}$. En un estudio fase II de CG avanzado con FOLFOX-4 en primera línea, las tasas de respuesta global fueron de 38\%, enfermedad estable 36\%, toxicidad hematológica grado 3/ $436 \%$ y neuropatía periférica grado 3 de $5 \% 16$. 
Tabla 4. Toxicidad grado 3 ó 4 observada en estudios randomizados de Q M T en cáncer gástrico avanzado

\begin{tabular}{|c|c|c|c|c|c|c|c|c|c|c|}
\hline Año & Revista & \multicolumn{2}{|c|}{$\begin{array}{l}\mathrm{N} \text { por cada } \\
\text { rama de tto. }\end{array}$} & Diarrea & Mucositis & $\begin{array}{l}\text { Tromboci- } \\
\text { topenia }\end{array}$ & Neutropenia & $\begin{array}{l}\text { Náuseas } \\
\text { vómitos }\end{array}$ & Alopecia & Muerte \\
\hline \multirow[t]{2}{*}{1991} & $\mathrm{JCO}^{6}$ & FAMc & NR & 17 & 1 & NR & NR & 74 & 20 & 2 \\
\hline & & FAMTX & NR & 26 & 10 & & & 63 & 24 & 1 \\
\hline \multirow[t]{2}{*}{1998} & Cancer $^{8}$ & EEP & 64 & 1,6 & 1,6 & 9,4 & 9,4 & 6,3 & 0 & NR \\
\hline & & FEC & 67 & 1,5 & 1,5 & 6 & 6 & 9 & 0 & \\
\hline \multirow[t]{2}{*}{2002} & Ann Oncol ${ }^{9}$ & PVIF & 125 & 4,3 & 6 & 1 & 2,9 & 4,3 & NR & 0 \\
\hline & & PVIF Mc & 129 & 5,0 & 6,7 & 1,9 & 5,6 & 3,3 & & 0 \\
\hline \multirow[t]{2}{*}{1997} & $\mathrm{JCO}^{10}$ & FEC & 126 & 6 & 6 & 4 & 36 & 17 & 56 & 1 \\
\hline & & FAMTX & 130 & 7 & 7 & 8 & 59 & 5 & 42 & 2 \\
\hline \multirow[t]{3}{*}{2000} & $\mathrm{JCO}^{11}$ & ELF & 132 & 5 & 3 & 2 & 39 & 7 & 27 & 0 \\
\hline & & $\mathrm{FP}$ & 134 & 6 & 12 & 9 & 35 & 26 & 16 & 1 \\
\hline & & FAMTX & 133 & 3 & 9 & 5 & 43 & 8 & 19 & 3 \\
\hline \multirow[t]{2}{*}{2002} & $\mathrm{JCO}^{12}$ & FEC & 289 & 6 & 5 & 4 & 32 & 11 & 59 & 0,34 \\
\hline & & $\mathrm{McCF}$ & 285 & 5 & 5 & 15 & 24 & 11 & 10 & 0,35 \\
\hline \multirow[t]{3}{*}{2003} & $\mathrm{JCO}^{13}$ & $\mathrm{~F}$ & 105 & 0 & 1 & 2 & 5 & 5 & 0 & 1 \\
\hline & & $\mathrm{FP}$ & 105 & 3 & 6 & 17 & 53 & 7,9 & 0 & 3,8 \\
\hline & & UFTMc & 70 & 0 & 1,5 & 29 & 37 & 10,6 & 0 & 1,4 \\
\hline \multirow[t]{2}{*}{2005} & Proc Am & FLI & 170 & 21,6 & 16,9 & NR & 52 & 9,0 & & 0,6 \\
\hline & Soc Clin & $\mathrm{FP}$ & 163 & 7,2 & 2,4 & & 25 & 4,8 & NR & 2,9 \\
\hline \multirow[t]{2}{*}{2006} & $\mathrm{JCO}^{14}$ & DCF & 227 & 19 & 21 & 8 & 82 & 14 & NR & 2,7 \\
\hline & & $\mathrm{FP}$ & 224 & 8 & 27 & 13 & 57 & 17 & & 4,5 \\
\hline
\end{tabular}

FAMc : 5-FU, adriamicina, mitomicina $\mathrm{C}$

FAMTX : 5-FU, adriamicina, metotrexato

FP : 5-FU, cisplatino

F : :5-FU

EEP : Etoposido, epirrubicina, cisplatino

FEC : 5-FU, epirrubicina, cisplatino

NR : No registrado

En nuestro estudio usando FOLFOX-4 encontramos una tasa de respuesta de 73\%, considerablemente mayor que en los estudios publicados previamente, esto puede explicarse sólo a sesgo debido al bajo número de pacientes o a que existen diferencias en la sensibilidad a esta combinación de drogas en nuestra población. El 20\% de los pacientes logra enfermedad estable sin progresión, lo que es similar a lo alcanzado por otros esquemas publicados ${ }^{16-19}$.

La toxicidad que se observó en este estudio fue similar a lo publicado con el esquema FLO y FOLFOX-4, pero inferior a FOLFOX-6, la toxicidad hematológica y no hematológica grado 3/4 fue
PVIF : 5-FU en infusión continua

PVIFMc : 5-FU en infusión continua, mitomicina $\mathrm{C}$

McCF : Mitomicina C, 5-FU, cisplatino

UFTMc : Uracilo tegafur, mitomicina $\mathrm{C}$

DCF : Docetaxel, 5-FU, cisplatino

FLI : 5-FU, leucovorina, irinotecan

$6,8 \%{ }^{16,18,19}$. No hubo muertes relacionadas al tratamiento. Los protocolos de estudios fase III más activos han mostrado entre $1 \%$ y $4,5 \%$ de mortalidad y aumento considerable de la toxicidad hematológica lo cual puede deteriorar la calidad de vida de los pacientes.

La duración de la respuesta es limitada, con un promedio de 5,5 meses, posterior a lo cual todos los pacientes progresan y fallecen a causa de la enfermedad, confirmando la agresividad del CG y su capacidad de lograr resistencia al tratamiento. La resistencia a la QMT ha sido evaluada en estudios de miles de genes con perfiles genéticos o microarray donde se detectaron 250 genes 
asociados a la resistencia a una droga y 8 genes asociados a multirresistencia ${ }^{21}$, lo que demuestra la complejidad de vías moleculares que condiciona este fenómeno.

Sin embargo, el conocimiento de los mecanismos de multirresistencia abre nuevas perspectivas terapéuticas usando terapias moleculares tendientes a revertir o sobrepasar la resistencia a drogas como el uso de ARN de interferencia especifico para MDR1, compuestos heterocíclicos y neferina, los cuales se han utilizado in vitro hasta ahora ${ }^{22-24}$.

La búsqueda de mejores respuestas en cáncer gástrico ha llevado a la incorporación de terapias moleculares como tratamiento único o asociado a QMT. Se ha utilizado inhibidores de tirosina kinasas como erlotinib que mostró respuestas en $11 \%$ y enfermedad estable en $11 \%{ }^{25}$. Las asociaciones con QMT que se han usado son: bevacizumab y irinotecan/cisplatino con respuestas de

\section{REFERENCIAS}

1. Globocan. International agency for research on cancer. 2002.

2. Medina E, Kaempffer A. Mortalidad por cáncer en Chile: consideraciones epidemiológicas. Rev Méd Chile 2001; 129: 1195-202.

3. Dicken BJ, Bigam DL, Cass C, Mackey JR, Joy AA, Hamilton SM. Gastric adenocarcinoma: review and considerations for future directions. Ann Surg 2005; 241: 27-39.

4. Glimelius B, Hoffman K, Haglund U, Nyren O, SJODEN PO. Initial or delayed chemotherapy with best supportive care in advanced gastric cancer. Ann Oncol 1994; 5: 189-90.

5. Wagner AD, Grothe W, Behl S, Kleber G, Grothey A, Haerting J et al. Chemotherapy for advanced gastric cancer. Cochrane Database Syst Rev 2005; 18: CD004064.

6. Wils JA, Klein HO, Wagener DJ, Bleiberg H, Reis H, Korsten F et Al. Sequential high-dose methotrexate and fluorouracil combined with doxorubicin. A step ahead in the treatment of advanced gastric cancer: a trial of the European Organization for Research and Treatment of Cancer Gastrointesti-
$66,7 \%$, matuzumab y epirrubicina/cisplatino/capecitabina con respuestas de $52 \%$, cetuximab y 5 FU/leucovorina/oxaliplatino con respuestas de 64\%. El impacto que estas terapias tendrán en la sobrevida aún se desconoce.

Actualmente no existe QMT estándar en segunda y tercera línea en CG por lo que estos esquemas activos y de baja toxicidad son una alternativa a evaluar en este ámbito.

En conclusión, la QMT con FOLFOX-4 tiene una alta tasa de respuesta, es un régimen de baja toxicidad y creemos debe ser evaluado en un estudio fase III.

\section{Agradecimientos}

Agradecemos en forma especial a nuestra Secretaria Académica, Sra. Nancy Lamig Hutt por su valioso aporte en la confección de este manuscrito. nal Tract Cooperative Group. J Clin Oncol 1991; 9: 827-31

7. Kim NK, Park YS, Heo DS, Suh C, Kim SY, Park KC ET AL. A phase III randomized study of 5fluorouracil and cisplatin versus 5-fluorouracil, doxorubicin, and mitomycin $\mathrm{C}$ versus 5-fluorouracil alone in the treatment of advanced gastric cancer. Cancer 1993; 71: 3813-8.

8. Icli F, Celik I, Aykan F, Uner A, Demirkazik A, Ozet A ET AL. A randomized Phase III trial of etoposide, epirubicin, and cisplatin versus 5-fluorouracil, epirubicin, and cisplatin in the treatment of patients with advanced gastric carcinoma. Turkish Oncology Group. Cancer 1998; 83: 2475-80.

9. Tebbutt NC, Norman A, Cunningham D, Iveson T, Seymour M, Hickish T et al. A multicentre, randomized phase III trial comparing protracted venous infusion (PVI) 5-fluorouracil (5-FU) with PVI 5-FU plus mitomycin $\mathrm{C}$ in patients with inoperable oesophago-gastric cancer. Ann Oncol 2002; 13: 1568-75.

10. Webb A, Cunningham D, Scarffe JH, Harper P, Norman A, Joffe JK et al. Randomized trial comparing epirubicin, cisplatin, and fluorouracil versus fluorouracil, doxorubicin, and methotrexa- 
te in advanced esophagogastric cancer. J Clin Oncol 1997; 15: 261-7.

11. Vanhoefer U, Rougier P, Wilke H, Ducreux MP, Lacave AJ, Van Cutsem E et al. Final results of a randomized phase III trial of sequential highdose methotrexate, fluorouracil, and doxorubicin versus etoposide, leucovorin, and fluorouracil versus infusional fluorouracil and cisplatin in advanced gastric cancer: A trial of the European Organization for Research and Treatment of Cancer Gastrointestinal Tract Cancer Cooperative Group. J Clin Oncol 2000; 18: 2648-57.

12. Ross P, Nicolson M, Cunningham D, Valie J, Seymour M, Harper P, Price T et al. Prospective randomized trial comparing mitomycin, cisplatin, and protracted venous-infusion fluorouracil (PVI 5-FU) With epirubicin, cisplatin, and PVI 5-FUin advanced esophagogastric cancer. J Clin Oncol 2002; 20: 1996-2004.

13. Ohtsu A, Shimada Y, Shirao K, Boku N, Hyodo I, SAITO H ET AL. Randomized phase III trial of fluorouracil alone versus fluorouracil plus cisplatin versus uracil and tegafur plus mitomycin in patients with unresectable, advanced gastric cancer: The Japan Clinical Oncology Group Study (JCOG9205). J Clin Oncol 2003; 21: 54-9.

14. Van Cutsem E, Moiseyenko VM, TJulandin S, Majlis A, Constenla M, Boni C et al. Phase III study of docetaxel and cisplatin plus fluorouracil compared with cisplatin and fluorouracil as first-line therapy for advanced gastric cancer: a report of the V325 Study Group. JCO 2006; 24: 4991-7.

15. Oнтsu A. Current status and future prospects of chemotherapy for metastasis gastric cancer. Gastric Cancer 2005; 8: 95-102.

16. De Vita F, Orditura M, Matano E, Bianco R, Carlomagno C, Infusino S et al. A phase II study of biweekly oxaliplatin plus infusional 5-fluorouracil and folinic acid (FOLFOX-4) as first-line treatment of advanced gastric cancer patients. $\mathrm{Br} \mathrm{J}$ Cancer 2005; 92: 1644-9.
17. Kim DY, Kim JH, Lee SH, Kim TY, Heo DS, BANG YJ ET AL. Phase II study of oxaliplatin, 5-fluorouracil and leucovorin in previously platinum-treated patients with advanced gastric cancer. Ann Oncol 2003; 14:383-7.

18. Louvet C, Andre T, Tigaud JM, Gamelin E, Douillard JY, Brunet R et al. Phase II study of oxaliplatin, fluorouracil, and folinic acid in locally advanced or metastatic gastric cancer patients. J Clin Oncol 2002; 20: 4543-8.

19. Al-Batran Se, Atmaca A, Hegewisch-Becker S, Jaeger D, Hahnfeld S, Rummel MJ et al. Phase II trial of biweekly infusional fluorouracil, folinic acid, and oxaliplatin in patients with advanced gastric cancer. J Clin Oncol 2004; 22: 658-63.

20. Andre T, Boni C, Mounedji-Boudiaf L, Navarro M, TABernero J, Hickish T et al. Oxaliplatin, fluorouracil, and leucovorin as adjuvant treatment for colon cancer. N Engl J Med 2004; 350: 2343-51.

21. Kang HC, Kim IJ, Park JH, Shin Y, Ku JL, Jung MS et AL. Identification of genes with differential expression in acquired drug-resistant gastric cancer cells using high-density oligonucleotide microarrays. Clin Cancer Res 2004; 10: 272-84.

22. GaO FL, Wang F, Wu JL, Le XP, Zhang QX. Screening effective sequences of small interfering RNAs targeting MDR1 gene in human gastric cancer SGC7901/VCR cells. Zhonghua Zhong Liu Za Zhi 2006; 28: 178-82.

23. Lage H, Aki-Sener E, Yalcin I. High antineoplastic activity of new heterocyclic compounds in cancer cells with resistance against classical DNA topoisomerase II-targeting drugs. Int J Cancer 2006; 119: 213-20.

24. CAO JG, TANG XQ, ShI SH. Multidrug resistance reversal in human gastric carcinoma cells by neferine. World J Gastroenterol 2004; 10: 3062-4.

25. Dragovich T, Mccoy S, Fenoglio-Preiser CM, Wang J, Benedetti JK, Baker AF et al. Phase II trial of erlotinib in gastroesophageal junction and gastric adenocarcinomas: SWOG 0127. J Clin Oncol 2006; 24: 4922-7. 